PII: S0735-1933(01)00330-X

\title{
EFFECT OF EFFECTIVE TISSUE CONDUCTIVITY ON THERMAL DOSE DISTRIBUTIONS OF LIVING TISSUE WITH DIRECTIONAL BLOOD FLOW DURING THERMAL THERAPY
}

\author{
Tzu-Ching Shih ${ }^{(1)}$, Hong-Sen Kou ${ }^{(1)}$, and Win-Li Lin ${ }^{(2)}$ \\ Department of Mechanical Engineering, Tatung University ${ }^{(1)}$ \\ Institute of Biomedical Engineering, National Taiwan University ${ }^{(2)}$ \\ Taipei, Taiwan, R.O.C \\ (E-mail: hskou@ttu.edu.tw or empire@ms5.url.com.tw)
}

(Communicated by J.P. Hartnett and W.J. Minkowycz)

\begin{abstract}
This study proposes a modified transient bioheat transfer equation based on combing the porous medium property and the scalar effective thermal conductivity equation in order to include the directional effect of blood flow. By applying the porous medium model to describe the collective behavior of the heat transfer in living tissue with many small blood vessels, an analytical solution can be obtained by Green's function. Simulation results quantitatively show that the blood perfusion rate, the averaged blood velocity, the porosity and the heating period are the crucial factors determining the distribution of thermal dose for thermal therapies. In addition, longer heating schennes induce dependencies of both the blood perfusion and the enhanced thermal conductivity on increased temperature.
\end{abstract}

(C) 2002 Elsevier Science Ltd

\section{Introduction}

Blood flow is a crucial factor determining the effectiveness of thermal therapy, both for hyperthermia alone, and combined with either radiation or chemotherapy. During the treatment, the dissipation of heat from heated tissues is carried out by convection through blood flow and also by conduction or diffusion processes. During thermal treatment, the effect of blood flow on temperature distribution in tissue is considered as the cooling due to both thermally significant large vessels and the smaller microvasculature during thermal treatment [1]. In addition, a model considering only the effect of thermally significant large blood vessels can result in the temperature distributions during thermal therapy [2]. 
The temperature distributions inside the heated tissues, generally controlled by heating modalities, are obtained by solving the bioheat transfer equation, as proposed by Pennes [3] in 1948. However, the major criticism for the conventional Pennes' model focused on the assumption that the heat transfer by blood flow occurs in a non-directional, heat sink- or source-like term. Weibaum et al. [4] and Chen and Holmes [5] attempted to formulate a more realistic bioheat equation, which would account for the thermally convective contribution of the vasculature, in addition to the heat sink-or source-like term originally proposed by Pennes.

Since there is little information concerning either the effect of blood flow direction on the size of the thermal lesions or the enhancement of effective thermal conductivity due to blood perfusion, especially for transient and three-dimensional calculations. This study investigates the effect of blood flow direction on thermal distribution within the heated tumor volume, and the effect of thermal conductivity behavior.

\section{Methods}

\section{Temperature Predication}

The blood-perfused tissue volume, including blood flow in microvascular bed character with the blood flow direction, contains many vessels and can be regarded as a porous structure consisting of a tumor tissue fully filled with blood. A heating target volume containing the capillary-like tumor vascular bed with blood flow direction is shown in Fig. 1. Herein the vascular volume in tissue is fully occupied with blood flow. In other words, the vascular volume is equal to the blood volume. For the tumor tissue, neglecting the metabolic heat production term, it is possible to simplify the Pennes bioheat transfer equation shown in Equation (1). The energy transport equation of blood flow is used here as shown in Equation (2).

$$
\begin{gathered}
\rho_{t} c_{i} \frac{\partial T_{t}}{\partial t}=k_{e f f}\left(\frac{\partial^{2} T_{t}}{\partial x^{2}}+\frac{\partial^{2} T_{t}}{\partial y^{2}}+\frac{\partial^{2} T_{L}}{\partial z^{2}}\right)-W_{b} c_{b}\left(T_{t}-T_{a}\right)+q_{t}(x, y, z, t) \\
\rho_{b} c_{b}\left(\frac{\partial T_{b}}{\partial t}+u \frac{\partial T_{b}}{\partial x}+v \frac{\partial T_{b}}{\partial y}+w \frac{\partial T_{b}}{\partial z}\right)=k_{b}\left(\frac{\partial^{2} T_{b}}{\partial x^{2}}+\frac{\partial^{2} T_{b}}{\partial y^{2}}+\frac{\partial^{2} T_{b}}{\partial z^{2}}\right)+q_{b}(x, y, z, t)
\end{gathered}
$$




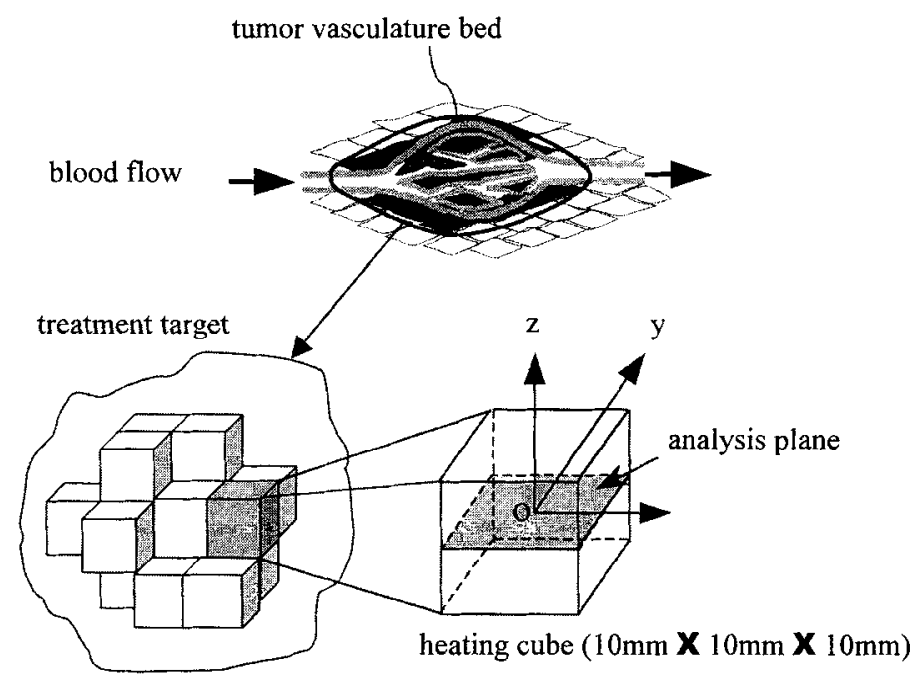

FIG. 1

Schematic illustration of the geometry of a heating cube studied for the distributions of th temperature and thermal dose equivalence $\left(E M_{43}\right)$ in the tissue. The initial temperatures of th selected heating cube and the surrounding tissue are set to $37^{\circ} \mathrm{C}$.

The overall effect on the energy transport can be estimated on the basis of the enhanced thermal conductivity in tissue, and $k_{e f f}$ is assigned to an equivalent value according to the experimental data of Crezze and Lagendijk [7] as

$$
k_{\text {eff }}=k_{t}\left(1+\beta W_{b}\right)
$$

where $\beta=0.02 \mathrm{~kg} / \mathrm{m}^{3} \mathrm{~s}$. Chen and Holmes [5] first showed that local thermal equilibrium could be achieved in very small vessels where their diameters are smaller or less than $0.2 \mathrm{~mm}$. In the present study, only the capillary network is included in the heated tissue. The volumetric averaging of the energy conservation principle is achieved by combining and rearranging equations (1) and (2) with the porous structure regarded as a homogeneous medium to obtain Equation (4).

$$
\begin{aligned}
& {\left[\phi \rho_{b} c_{b}+(1-\phi) \rho_{t} c_{t}\right] \frac{\partial T}{\partial t}+\phi \rho_{b} c_{b}\left[u \frac{\partial T}{\partial x}+v \frac{\partial T}{\partial y}+w \frac{\partial T}{\partial z}\right]} \\
& =\left[\phi k_{b}+(1-\phi) k_{e f f}\right]\left(\frac{\partial^{2} T}{\partial x^{2}}+\frac{\partial^{2} T}{\partial y^{2}}+\frac{\partial^{2} T}{\partial z^{2}}\right)-(1-\phi) W_{b} c_{b}\left(T-T_{a}\right)+(1-\phi) q_{t}+\phi q_{b}
\end{aligned}
$$


The porosity, $\phi$, is defined as the ratio of blood volume to the total volume. During the simulation, the ultrasound power was uniformly deposited within the desired heating volume and the absorption of ultrasound power by the blood is assumed to be $10 \%$ of that for the tissue [9]. The dimensionless parameters have the following forms:

$$
X=\frac{x}{l}, Y=\frac{y}{l}, Z=\frac{z}{l}, T^{*}=\frac{T-T_{\alpha}}{T_{a}}, \tau=\frac{\phi k_{b}+(1-\phi) k_{\text {eff }}}{\left[\phi \rho_{b} c_{b}+(1-\phi) \rho_{t} c_{t}\right] l^{2}} t
$$

by setting the product-form as

$$
\mathrm{T}^{*}(X, Y, Z, \tau)=\bar{T}(X, Y, Z, \tau) \exp \left(c_{1} X+c_{2} Y+c_{3} Z-c_{4} \tau\right)
$$

where the coefficients have the forms:

$$
\begin{aligned}
& c_{1}=\frac{\phi u l}{2 \alpha_{e f f}}, c_{2}=\frac{\phi v l}{2 \alpha_{e f f}}, c_{3}=\frac{\phi w l}{2 \alpha_{e f f}}, \gamma=\frac{(1-\phi) W_{b} c_{b} l^{2}}{\phi k_{b}+(1-\phi) k_{e f f}} \\
& c_{4}=c_{1}{ }^{2}+c_{2}{ }^{2}+c_{3}{ }^{2}+\gamma, \alpha_{e j f}=\frac{\phi k_{b}+(1-\phi) k_{e f f}}{\rho_{b} c_{b}}
\end{aligned}
$$

Substituting Equations (5) and (6) into Equation (4), Equation (4) can be expressed in dimensionless form as:

$$
\frac{\partial \bar{T}}{\partial \tau}=\frac{\partial^{2} \bar{T}}{\partial X^{2}}+\frac{\partial^{2} \bar{T}}{\partial Y^{2}}+\frac{\partial^{2} \bar{T}}{\partial Z^{2}}+q_{1}(X, Y, Z, \tau)
$$

where

$$
q_{1}(X, Y, Z, \tau)=\frac{\left.\left[\phi q_{b}+(1-\phi) q_{1}\right]\right]^{2}}{T_{a}\left[\phi k_{b}+(1-\phi) k_{c f f}\right]} \times \exp \left[-\left(c_{1} X+c_{2} Y+c_{3} Z-c_{4} \tau\right)\right]
$$

For an infinite medium $(-\infty<X<\infty,-\infty<Y<\infty, \quad-\infty<Z<\infty)$ and for time $0<\tau$, the desired Green's function [10] is not difficult to find, and is given by

$$
G\left(X, Y, Z, \tau \mid X_{p}, Y_{p}, Z_{p}, \tau_{p}\right)=\left[4 \pi\left(\tau-\tau_{p}\right)\right]^{-\frac{3}{2}} \exp \left[-\frac{\left(X-X_{p}\right)^{2}+\left(Y-Y_{p}\right)^{2}+\left(Z-Z_{p}\right)^{2}}{4\left(\tau-\tau_{p}\right)}\right]
$$

where the positions and time with the subscript $p$ refer to a heating power location. Green's function $G\left(X, Y, Z, \tau \mid X_{p}, Y_{p}, Z_{p}, \tau_{p}\right)$ represents the dimensionless temperature $\bar{T}(X, Y, Z, \tau)$, at the 
location $(X, Y, Z)$, and at time $\tau$, due to an instantaneous heat source located at $\left(X_{p}, Y_{p}, Z_{p}\right)$ releasing its energy spontaneously at time $\tau_{p}$. The solution of Equation (8) can be written in terms of the above Green's function as

$$
\begin{aligned}
T\left(X, Y, Z, \tau \mid X_{p}, Y_{p}, Z_{p}, \tau_{p}\right)= & \int_{\tau_{s}}^{\tau_{f}} \int_{X_{,}}^{X_{j}} \int_{Y_{s}}^{Y_{f}} \int_{Z_{s}}^{Z_{f}} G\left(X, Y, Z, \tau \mid X_{p}, Y_{p}, Z_{p}, \tau_{p}\right) \\
& \times q_{1}\left(X_{p}, Y_{p}, Z_{p}, \tau_{p}\right) \\
& \times d Z_{p} d Y_{p} d X_{p} d \tau_{p}
\end{aligned}
$$

where the positions and time with the subscripts $s$ and $f$ refer to the start and finish of the computational domain, respectively. Finally, the transient and three-dimensional analytical solution of Equation (4) in terms of the dimensionless form can be written as:

$$
T(x, y, z, t)=T_{a}\left\{1+\bar{T}\left(X, Y, Z, \tau \mid X_{p}, Y_{p}, Z_{p}, \tau_{p}\right) \exp \left(c_{1} X+c_{2} Y+c_{3} Z-c_{4} \tau\right)\right\}
$$

The approximate solution of Equation (12) can be evaluated by the Gauss-Quadrature integration scheme.

\section{Thermal Dose Calculation}

The thermal dose or equivalent minutes at $43^{\circ} \mathrm{C}$ defined by Sapareto and Dewey [11] can describe the extent of thermal damage or destruction of tissue, and its expression is used as following:

$$
E M_{43}(\text { in } \min .)=\int R^{\left(T^{\prime}-43\right)} d t
$$

where $R=2$ for $T \geq 43^{\circ} \mathrm{C}, R=4$ for $37^{\circ} \mathrm{C}<T<43^{\circ} \mathrm{C}$, $\mathrm{T}$ is temperature, and $t$ is time. The threshold dose for necrosis ranges from $E M_{43}=25$ to $240 \mathrm{~min}$ for brain and muscle tissue, respectively [11,12]. In this simulation study, a conservative value $\left(E M_{43}=300\right)$ has been taken. Parameter values of simulations are shown in Table 1. 
TABLE 1

Parameters used for Simulation

\begin{tabular}{|c|c|c|c|}
\hline Symbol & Definition & Value & Units \\
\hline$\rho_{t}, \rho_{b}$ & Density of tissue and blood & 1050 & $\mathrm{~kg} / \mathrm{m}^{3}$ \\
\hline$c_{t}, c_{b}$ & Specific heat of tissue and blond & 3770 & $\mathrm{~J} / \mathrm{kg}{ }^{\circ} \mathrm{C}$ \\
\hline$k_{t}, k_{b}$ & Thermal conductivity of tissue and blood & $0.5-4.8[7]$ & $\mathrm{W} / \mathrm{m}^{\circ} \mathrm{C}$ \\
\hline$u$ & Average blood velocity & $0.3-2[6-7]$ & $\mathrm{mm} / \mathrm{s}$ \\
\hline$W_{b}$ & Blood perfusion rate & $0.5-20[8]$ & $\mathrm{kg} / \mathrm{m}^{3} \mathrm{~s}$ \\
\hline$T_{a}$ & Arterial temperature & 37 & ${ }^{\circ} \mathrm{C}$ \\
\hline
\end{tabular}

\section{Results and Discussion}

Figures 2(a) to 2(d) show the transient temperature and thermal dose profiles at the origin inside the heated cube, as shown in Fig. 1. Figures 2(a) and 2(b) illustrated that the temperature increases during the heating duration and then decays after the heating power is turned off. It was observed that the resulted peak temperatures of rapid heating for case III are higher than those of long heating for case IV, as shown in Table 2. Rapid heating schemes induce almost the same peak temperature values, even when $k_{t}$ changed from 0.6 to $4.8 \mathrm{~W} / \mathrm{m}^{\circ} \mathrm{C}$, as shown in Fig. 2(a). But for longer heating schemes, the peak temperatures decreased as the tissue conductivity values increased, especially considering the enhanced thermal tissue conductivity effect $\left(\beta=0.02 \mathrm{~kg} / \mathrm{m}^{3} \mathrm{~s}\right)$ due to blood flow. Under the same total input energy $\left(91 \mathrm{~W} / \mathrm{cm}^{3}\right)$, the peak temperature increases with the shorter period of heating. A higher peak temperature can yield a higher thermal dose level, as shown in Figs. 2(c) and 2(d). It is clear that the accumulations of $E M_{43}$ levels depend mainly on the peak temperature. In addition, the influence of enhanced thermal conductivity on peak temperature in tissue due to blood flow is not significant for rapid heating. Consequently, the above results show that the temperature distribution within the heated tissue depends on both the heating scheme and the enhanced thermal conductivity of tissue during thermal therapy.

TABLE 2

Values of the two different heating schemes

\begin{tabular}{|c|c|c|c|c|c|c|}
\hline Case & I & II & III & IV & V & VI \\
\hline Heating power density $q\left(\mathrm{~W} / \mathrm{cm}^{3}\right)$ & 50 & 2 & 50 & 2 & 50 & 2 \\
\hline Absorbed power density $q_{a}=(1-0.9 \phi) q\left(\mathrm{~W} / \mathrm{cm}^{3}\right)$ & 47.75 & 1.91 & 45.5 & 1.82 & 41 & 1.64 \\
\hline Heating duration $(\mathrm{s})$ & 2 & 50 & 2 & 50 & 2 & 50 \\
\hline Porosity & \multicolumn{2}{|c|}{$\phi=0.05$} & $\phi=0.1[8]$ & \multicolumn{2}{|c|}{$\phi=0.2$} \\
\hline
\end{tabular}



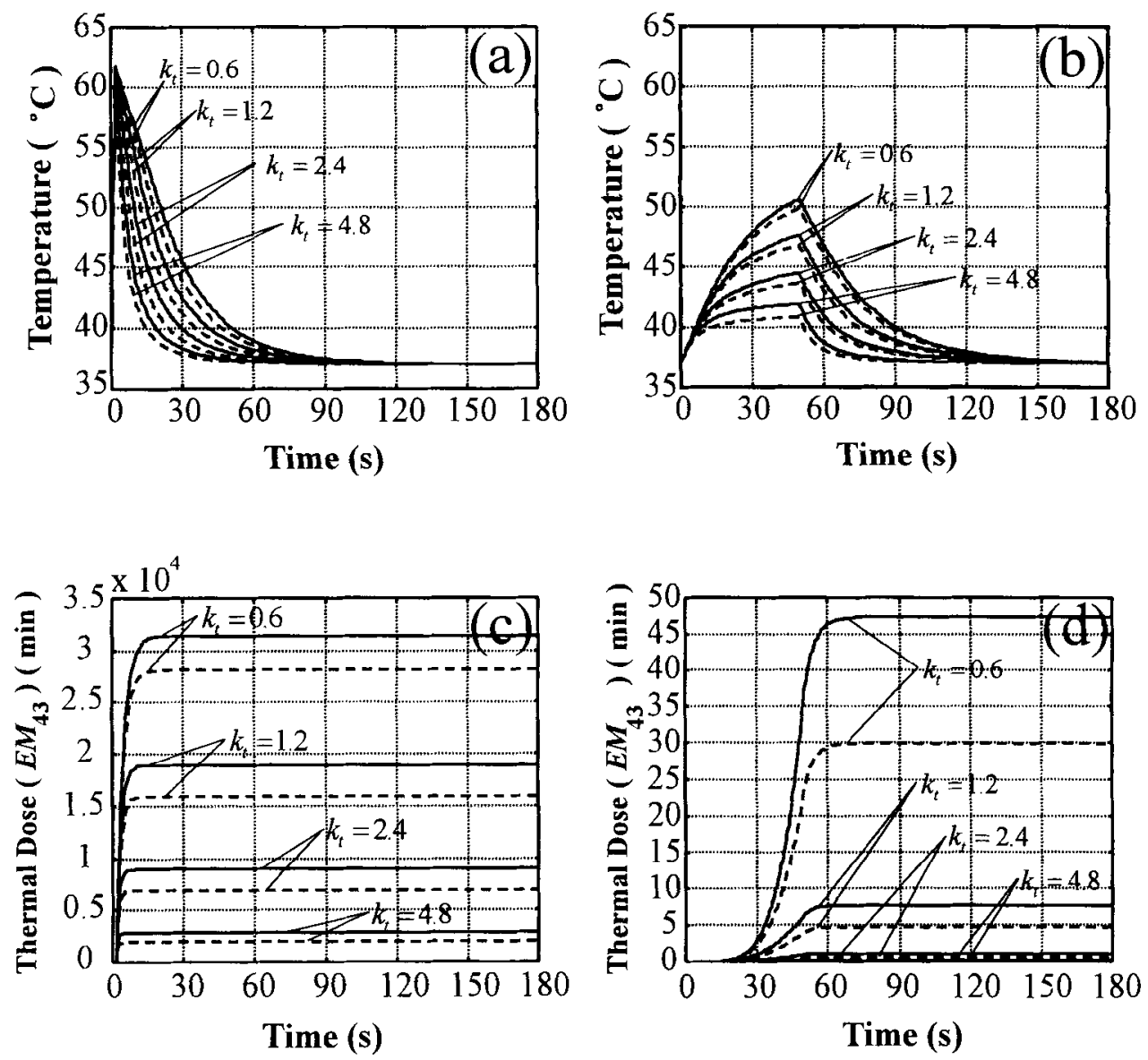

FIG. 2

Effects of four different tissue conductivity values, two different $\beta$ values and two different heating schemes on the transient temperature and thermal dose profiles for $W_{\mathrm{b}}=10 \mathrm{~kg} / \mathrm{m}^{3} \mathrm{~s}, \phi=0.1$ $u=1 \mathrm{~mm} / \mathrm{s}, \quad k_{b}=0.6 \mathrm{~W} / \mathrm{m}^{\circ} \mathrm{C}$, and with $k_{t}$ ranging from 0.6 to $4.8 \mathrm{~W} / \mathrm{m}{ }^{\circ} \mathrm{C}$ [7]. The solid lines represent $\beta=0$, and the dashed lines represent $\beta=0.02$, (a)-(c) for case III; (b)-(d) for case IV. 

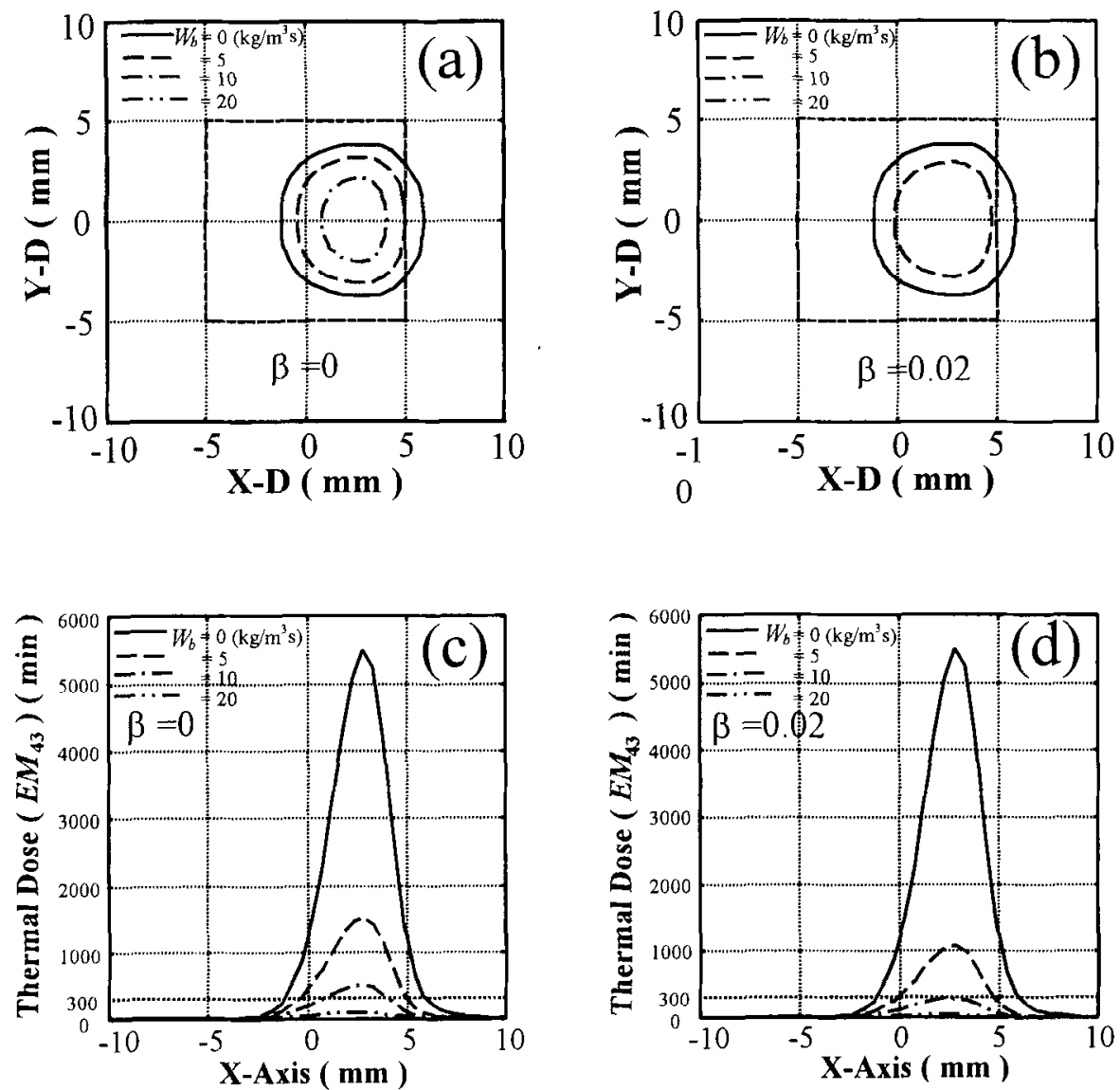

FIG. 3

Effects of blood perfusion rates with two different $\beta$ values on thermal distributions at $t=180 \mathrm{~s}$ for the heating case IV in Table 2, for $\phi=0.1, u=1 \mathrm{~mm} / \mathrm{s}, k_{b}=k_{t}=0.5 \mathrm{~W} / \mathrm{m}{ }^{\circ} \mathrm{C}$, (a)-(b) for $E M_{43}=300$ min contours; (c)-(d) the corresponding thermal dose along the $\mathrm{X}$-axis. 

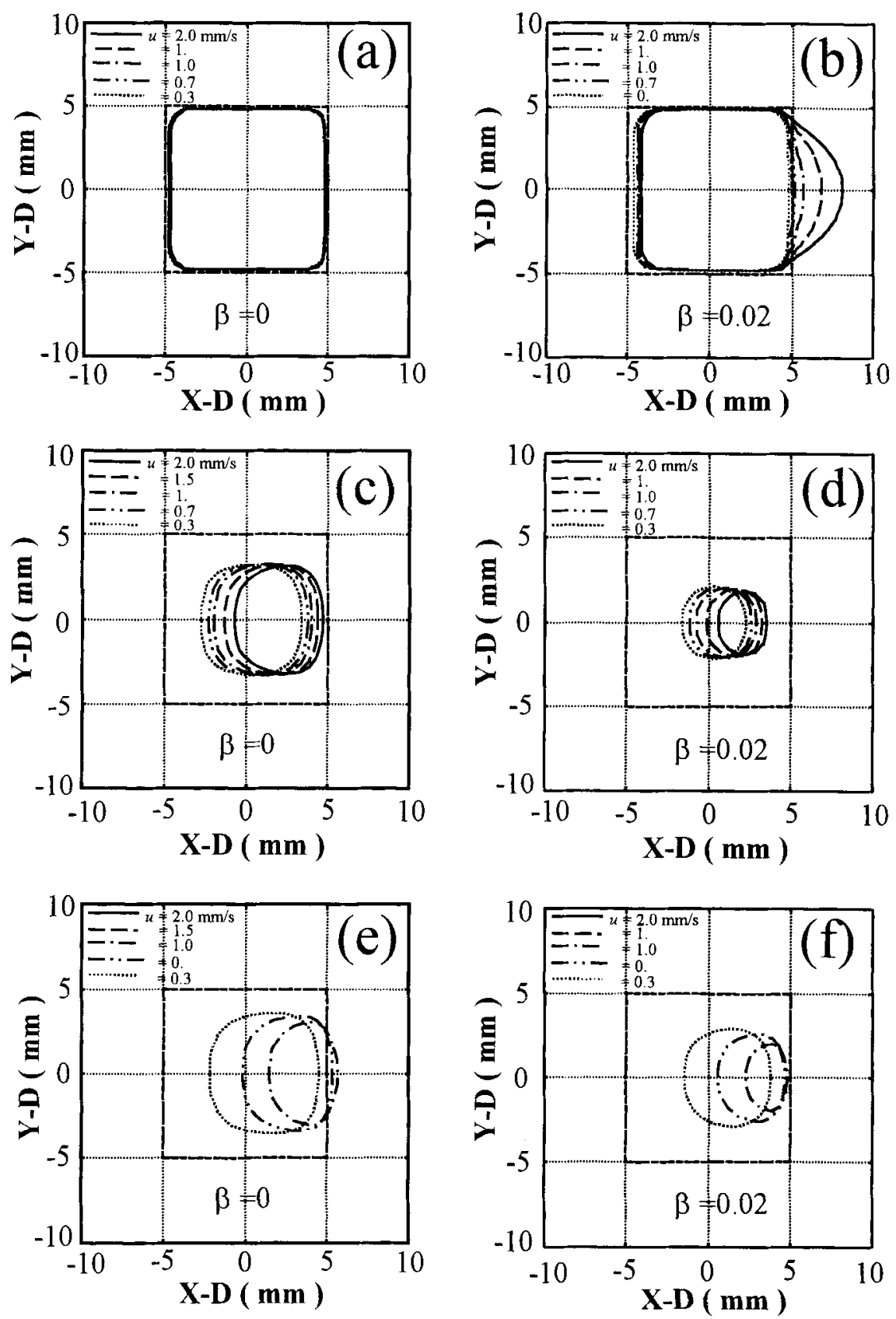

FIG. 4

Effects of variations of porosity and velocity on thermal dose contours at $t=180 \mathrm{~s}$ $W_{b}=20 \mathrm{~kg} / \mathrm{m}^{3} \mathrm{~s}, u$ ranging from 0.3 to $2 \mathrm{~mm} / \mathrm{s}$, (a)-(b) for $E M_{43}=300 \mathrm{~min}$ contours with heating cases I and V; (c)-(d) and (e)-(f) for $E M_{43}=25$ min contours with heating cases II an VI, respectively; (a)(c)(d) with $\phi=0.05$; (b)(e)(f) with $\phi=0.2$. 
Figures 3(a) and (b) show that the thermal dose equivalence for contours $E M_{43}=300 \mathrm{~min}$ inside the treatable region cover the wanted therapeutic region, but they exceed the heating target downstream from the normal tissue with lower blood perfusion rates at $t=180 \mathrm{~s}$ in case IV with longer heating. The thermal lesion dimensions are clearly decreased as the perfusion rate increases, as shown in Figs. 3 (c) and 3 (d). Comparing $\beta=0$ and $\beta=0.02 \mathrm{~kg} / \mathrm{m}^{3} \mathrm{~s}$, when considering the enhanced thermal effect, the thermal dose $E M_{43}=\mathbf{3 0 0}$ min contours did not exist, as shown in Fig. 3(b). Figure 3 shows that a higher perfusion rate has a lower thermal dose level. A higher perfusion rate leads to not only smaller thermal lesion region but also lower thermal dose levels. These results also agree with those obtained by Kolios et al. [2]. The perfusion effect can act as a heat sink to remove the deposition energy, and hence to prevent the blood flow from transporting the input energy downstream of the normal tissue.

The rapid heating is effective in delivering the power energy to the desired treatment volume during thermal therapy only when the porosity is small and the average blood velocity is low, as shown in Fig. 4 (a). When the porosity value reaches 0.2 and $\beta$ equals $0.02 \mathrm{~kg} / \mathrm{m}^{3} \mathrm{~s}$, the region of thermal lesion still effectively covers the wanted therapeutic region, but it exceeds the heating target downstream from the normal tissue, as shown in Fig. 4 (b). For longer heating, however, the regions enclosed by contours for $E M_{43}=25$ min with the effective conductivity model are smaller than those without it, as shown in Figs. $4(c)$ to $4(f)$. Consequently, it is clear that higher values of porosity and velocity induce larger areas of overheating downstream from normal tissue.

\section{Conclusions}

- Heat transfer in living tissue, including blood flow in microvascular bed, can be modeled by solving a conjugate problem with the porous medium concept.

- An analytical solution of the porous medium model can be obtained by means of Green's function.

- The scalar effective thermal conductivity behavior must be taken into account for longer heating.

- The peak temperature decreases with the increase of heating duration and during thermal therapy it dominates the thermal dose level of delivering input energy.

- Higher perfusion rates lead to both smaller thermal lesion regions and also lower thermal dose levels.

- Blood flow has the potential of transporting the deposition energy from the heated region target to downstream from the surrounding normal tissues, especially with high velocity and porosity. 


\section{Nomenclature}

$c$ specific heat $\left(\mathrm{J} / \mathrm{kg}{ }^{\circ} \mathrm{C}\right)$

$k$ thermal conductivity $\left(\mathrm{W} / \mathrm{m}^{\circ} \mathrm{C}\right)$

$k_{\text {eff }}$ scalar effective thermal conductivity $\left(\mathrm{W} / \mathrm{m}^{\circ} \mathrm{C}\right)$

$l$ characteristic length $(\mathrm{mm})$

$t$ time (s)

$T$ temperature $\left({ }^{\circ} \mathrm{C}\right)$

$T_{a}$ arterial temperature $=37\left({ }^{\circ} \mathrm{C}\right)$

$T^{*}$ nondimensional temperature $T^{*}=\frac{T-T_{a}}{T_{a}}$

$q$ heat power density $\left(\mathrm{W} / \mathrm{cm}^{3}\right)$

$q_{a}$ absorbed power density $q_{a}=(1-0.9 \phi) q\left(\mathrm{~W} / \mathrm{cm}^{3}\right)$

$u$ average blood velocity along $\mathrm{x}$-directional $(\mathrm{mm} / \mathrm{s})$

$v$ average blood velocity along y-directional $(\mathrm{mm} / \mathrm{s})$

$w$ average blood velocity along z-directional $(\mathrm{mm} / \mathrm{s})$

$x$ distance along $\mathrm{x}$-axis ( $\mathrm{mm}$ )

$y$ distance along $\mathrm{y}$-axis ( $\mathrm{mm}$ )

$z$ distance along z-axis ( $\mathrm{mm}$ )

$X$ nondimensional $X=x / l$

$Y$ nondimensional $Y=y / l$

$Z$ nondimensional $Z=z / l$

$W_{b}$ blood perfusion rate $\left(\mathrm{Kg} / \mathrm{m}^{3} \mathrm{~s}\right)$

$\phi$ ratio of blood volume to the total volume

$\beta$ coefficient constant $\left(\mathrm{kg} / \mathrm{m}^{3} \mathrm{~s}\right)$

$\rho$ density $\left(\mathrm{kg} / \mathrm{m}^{3}\right)$

$\tau$ nondimensional time $\tau=\frac{\phi k_{b}+(1-\phi) k_{e f t}}{\left[\phi \rho_{b} c_{b}+(1-\phi) \rho_{l} c_{t}\right] l^{2}} t$

\section{Subscripts}

$b$ blood 
$p$ power

$t$ tissue

\section{$\underline{\text { References }}$}

1. J. W. Baish, ASME J. Biomech. Eng. 116, 521 (1994).

2. M. C. Kolios, M. D. Sherar, and J. W. Hunt, Med. Phys. 23, 1287 (1996).

3. H. H. Pennes, J. Appl. Physiol. 1, 93 (1948).

4. S. Weinbaum, L. Jiji, and D. E. Lemons, ASME J. Biomech. Eng. 106, 312 (1984).

5. M. M. Chen, and K. R. Holmes, Ann. N.Y. Acad. Sci. 335, 137 (1980).

6. A.C. Guyton, Textbook of Medical Physiology. 7th ed., Saunders, Philadelphia, pp. 218-220 (1986).

7. J. Crezee, and J. J. Lagendijk, Phys. Med. Biol. 37, 1321 (1992).

8. D. E. Hilmas, and E. L. Gillette, Cancer J. 33, 103 (1974).

9. F. A. Duck, Physical Properties of Tissues: A Comprehensive Reference Book, Academic Press, San Diego (1990).

10. M. N. Özisik, Heat Conduction, 2nd ed., John Wiley \& Sons, New York (1993).

11. S. A. Sapareto, and W. Dewey, Int. J. Radiat. Oncol. Biol. Phys. 10, 787 (1984).

12. C. Damianou, and K. Hynynen, Ultrasound Med. Biol. 19, 777 (1993).

Received October 15, 2001 